\title{
Variação genética em caracteres de crescimento em progênies de Dipteryx alata Vog.
}

\author{
Genetic variation in growth traits \\ of Dipteryx alata Vog progenies
}

\author{
Maximiliano Kawahata Pagliarini' ${ }^{1}$, Eliana Cristina Generoso Konrad ${ }^{2}$, \\ Flaviana Cavalcanti da Silva ${ }^{1}$, Mayte Sarmpaio Cesário da Silva ${ }^{3}$, \\ Juliana Prado Moreira1, Aida Sanae Sato ${ }^{4}$, José Arimatéia Rabelo Machado ${ }^{5}$, \\ Miguel Luiz Menezes Freitas ${ }^{6}$, Ananda Virgínia Aguiar ${ }^{7}$, \\ Mário Luiz Teixeira de Moraes $^{8}$, Alexandre Magno Sebbenn ${ }^{9}$
}

\begin{abstract}
Resumo
O objetivo desse trabalho foi estimar a variação genética entre e dentro de progênies de Dipteryx alata, com a finalidade de realizar um desbaste seletivo, visando estabelecer um pomar de sementes por mudas com variabilidade e qualidade genética. O ensaio foi instalado em dezembro de 1986, na Estação Experimental de Pederneiras, no Florestal Estadual de São Paulo, seguindo-se o delineamento de blocos ao acaso, com 25 progênies, cinco repetições e cinco plantas por parcela. O espaçamento utilizado foi o de $3 \times 3$ m. O teste de progênies foi avaliado aos 25 anos para os caracteres: altura total, diâmetro à altura do peito (DAP), volume real, bifurcação e sobrevivência. Foram realizadas análises de deviance para determinar a variação genética entre e dentro de progênies para os caracteres. As estimativas de componentes de variância e parâmetros genéticos foram obtidas pelo método REML/BLUP (máxima verossimilhança restrita - melhor predição linear não viciada), empregando-se o software genético-estatístico Selegen. Pelo teste da razão de verossimilhança não foram detectadas diferenças significativas entre e dentro de progênies de Dipteryx alata para os caracteres de crescimento, apenas para bifurcação observou-se diferença significativa tanto para o efeito de progênies quanto para o efeito de parcela. As herdabilidades individuais no sentido restrito foram muito baixas para maioria dos caracteres, com exceção da altura $(0,27)$, que por sua vez, apresentou a maior acurácia. Recomenda-se realizar desbaste para eliminar os indivíduos de baixa produção e aumentar a base genética dessa população a partir da introdução de outros genótipos.
\end{abstract}

Palavras-chave: conservação genética, herdabilidade, produção de sementes florestais, recursos genéticos florestais, variabilidade genética.

\begin{abstract}
The aim of this work was to estimate the genetic variation among and within Dipteryx alata progenies to propose a selective logging to establish a seedling seed orchard with variability and genetic quality. The progeny test was established in December 1986 at Pederneiras Experimental Station, São Paulo Forestry Institute, following a randomised block design with 25 progenies, five replications, five plants per plot and
\end{abstract}

1Doutorando em Agronomia. UNESP - Universidade Estadual Paulista Júlio de Mesquita Filho / Faculdade de Engenha-
ria de Ilha Solteira. Av. Brasil 56 - Centro - 15385000 - Ilha Solteira, SP, Brasil. E-mail: maxpagliarini@hotmail.com;
flaviana cavalcanti@ @otmail.com; julianapmbio@yahoo.com.br. 2Professor Doutor. Faculdades Adamantinense Integradas. Centro Universitário de Adamantina. Rua 9 de Julho, 730 Centro - 17800000 - Adamantina, SP, Brasil. E-mail: eliana generoso@bol.com.br.

${ }^{3}$ Mestrado em andamento em Engenharia Florestal. UFPR - Universidade Federal do Paraná. Av. Pref. Lothario Meissner, 3400 - Jardim Botânico - 80210-170 - Curitiba, PR - Brasil. E-mail: mahgtm@ hotmail.com.

${ }^{4}$ Pesquisadora Florestal. IF - Instituto Florestal do Estado de São Paulo, Divisao de Dasonomia / Secao de Silvicultura. Rua do Horto - Horto Florestal - 02377000 - São Paulo, SP, Brasil. E-mail: aidasato@if.sp.gov.br.

${ }^{5}$ Pesquisador Doutor. IF - Instituto Florestal do Estado de São Paulo, Divisao de Florestas e Estacoes Experimentais. Avenida Rodrigues Alves, 38-25 - Horto Florestal - 17030-000 - Bauru, SP - Brasil. E-mail: j.rabelomachado@yahoo.com.br.

${ }^{6}$ Pesquisador Doutor. IF - Instituto Florestal do Estado de São Paulo, Divisao de Dasonomia / Secao de Silvicultura. Rua do Horto - Horto Florestal - 02377000 - São Paulo, SP, Brasil. E-mail: miguellmfreitas@yahoo.com.br.

${ }^{7}$ Pesquisador Doutora. Embrapa Florestas. Estrada da Ribeira, km 111 - Caixa Postal - 83411000 - Colombo, PR, Brasil. E-mail: ananda.aguiar@embrapa.br.

${ }^{8}$ Professor Titular. UNESP - Universidade Estadual Paulista Júlio de Mesquita Filho / Faculdade de Engenharia de Ilha Solteira. Av. Brasil 56 - Centro - 15385000 - Ilha Solteira, SP, Brasil. E-mail: teixeira@agr.feis.unesp.br.

${ }^{9}$ Pesquisador Doutor. IF - Instituto Florestal de São Paulo / Estação Experimental de Tupi. Rodovia Luiz de Queiroz, Km 149,5 - Caixa-postal: 339 - Tupi - 13400-970 - Piracicaba, SP, Brasil. E-mail: alexandresebbenn@yahoo.com.br.

Sci. For., Piracicaba, v. 44, n. 112, p. 925-935, dez. 2016 DOI: dx.doi.org/10.18671/scifor.v44n112.14 
$3 \times 3 \mathrm{~m}$ spacing. The test was measured 25 years after planting for the traits: total height, diameter at breast height $(\mathrm{DBH})$, wood volume, forking and survival. Deviance analyses were performed to determine the genetic variation among and within progenies for growth traits. The variance components and genetic parameters estimates were obtained by REML/BLUP methods (Restricted maximum likelihood/Best linear unbiased prediction) using the Selegen software. Based on likelihood ratio test, no significant differences between and within Dipteryx alata progenies were detected to growth traits, only for forking there was a significant difference in relation to progeny and plot. The individual narrow sense heritability was very low for most of the traits, except for the height (0.27), which in turn, had the highest accuracy. It is recommended to perform a selective logging to eliminate low production trees and increase the genetic basis of this population from the introduction of other genotypes.

Keywords: genetic conservation, heritability, forest seed production, forest genetic resources, genetic variability.

\section{INTRODUÇÃO}

A longa duração e os expressivos investimentos necessários para transformar um componente da biodiversidade em um recurso genético de valor econômico impedem que uma fração maior de espécies florestais nativas do Brasil possa ser prospectada visando a maior adaptação às necessidades humanas (CLEMENT, 2001; CLEMENT et al., 2005). A domesticação de novas espécies não é uma atividade típica e corriqueira, depende principalmente do aprimoramento das práticas silviculturais e da seleção de material genético mais apropriado.

Os biomas brasileiros têm grande número de espécies promissoras que poderiam, a partir de técnicas sustentáveis, ser prospectadas. É o caso do Cerrado - segundo maior bioma brasileiro, ocupando $21 \%$ do território nacional e considerado a última fronteira agrícola do planeta (BORLAUG, 2002). É uma das áreas de maior biodiversidade no mundo (MITTERMEIER et al., 2004), sendo, inclusive, destacado como um hotspot da conservação da biodiversidade (MYERS et al., 2000).

Entre as espécies da flora do cerrado, Dipteryx alata Vog. (baru) se destaca devido ao seu potencial de uso tanto para produção de madeira como para alimentação animal e humana, sua amplitude de ocorrência, o relevante potencial econômico e as características silviculturais que favorecem o seu uso em sistemas agroflorestais (BOTEZELLI et al., 2000). Caracteres de crescimento e os relacionados à produção de frutos deveriam ser considerados no processo de melhoramento já que a composição de suas sementes e frutos é utilizada nas indústrias alimentícia, oleoquímica (TAKEMOTO et al., 2001; OLIVEIRA, 1998) e farmacêutica (CZEDER, 2009).

Assim como toda espécie não domesticada, Dipteryx alata apresenta variabilidade genética, indicando que essa espécie é promissora para o melhoramento genético (ROCHA et al., 2002). Porém, de nada adianta essa potencialidade se não houver políticas de conservação genética das populações remanescentes, já que o mesmo pode desaparecer com a frequente devastação do Cerrado. Esse bioma, desde a década de 1970, vem sofrendo acelerado processo de degradação a partir da expansão agropecuária, já que técnicas agrícolas transformaram seus solos antes impróprios em terras muito produtivas (SANTOS et al., 2014a). Além disso, a conservação das espécies nativas e, de maneira geral, dos recursos naturais como um todo serve para manter a representatividade dessas espécies, conservar os recursos naturais para amenizar catástrofes humanas e ambientais, conservar a máxima variabilidade genética e formar corredores ecológicos, sendo seu uso uma das alternativas viáveis à conservação dos recursos genéticos. Diversas espécies florestais são fonte de matéria prima para várias finalidades. Porém, para sua utilização é necessário conhecimento do potencial genético e produtivo (KUBOTA et al., 2015).

O presente trabalho teve como objetivo estimar a variação genética entre e dentro de progênies de D. alata para propor um desbaste seletivo visando estabelecer um pomar de sementes por mudas para produção de sementes com variabilidade e qualidade genética.

\section{MATERIAL E MÉTODOS}

\section{Amostragem e delineamento experimental}

As plantas avaliadas neste trabalho fazem parte de uma coleção de germoplasma instalada no município de Pederneiras, SP, com sementes procedentes do município de Icém, SP. O teste de 
progênies foi implantado em dezembro de 1986, na Floresta Estadual de Pederneiras $\left(22^{\circ} 21^{\prime} 08^{\prime \prime} \mathrm{S}\right.$, $48^{\circ} 46^{\prime} 31^{\prime \prime} \mathrm{O}$, altitude de $475 \mathrm{~m}$ ). O local apresenta verão quente e úmido e inverno seco, temperatura anual oscilando entre 16 e $34^{\circ} \mathrm{C}$ e precipitação pluviométrica média anual de $1.300 \mathrm{~mm}$. O teste foi estabelecido utilizando o delineamento experimental de blocos completos ao acaso, com 25 tratamentos (progênies), cinco repetições e cinco plantas por parcela. O espaçamento utilizado foi $3 \times 3 \mathrm{~m}$.

Aos 25 anos de idade todas as plantas do teste foram avaliadas quanto aos seguintes caracteres: altura total $(\mathrm{h}, \mathrm{em} \mathrm{m})$; diâmetro à altura do peito $(\mathrm{DAP}, \mathrm{em} \mathrm{cm})$; volume real $\left(\mathrm{Vol}, \mathrm{m}^{3}\right.$ planta $\left.^{-1}\right)$; bifurcação total (Bif), sendo que, neste caso, foram atribuídas notas às plantas variando de 1 a 12 (1= plantas com 12 bifurcações, ..., 12 = plantas com nenhuma bifurcação) e sobrevivência. $O$ volume $\left(\mathrm{V}_{c}\right)$, foi calculado conforme a expressão $V_{c}=\left(\pi D A P^{2} / 40000\right) h$.

\section{Estimativa de componentes da variância e parâmetros genéticos}

Os caracteres quantitativos foram analisados usando-se o modelo linear misto (modelo aditivo univariado), aplicando o procedimento de melhor preditor linear não viciado REML/BLUP (máxima verossimilhança restrita/melhor predição linear não viciada) do software genético-estatístico Selegen desenvolvido por Resende (2002b). No modelo adotado considerou os seguintes efeitos: progênies de meios-irmãos, blocos completos ao acaso, várias plantas por parcela, um só local e uma única população, sendo $\mathrm{y}=\mathrm{Xr}+\mathrm{Za}+\mathrm{Wp}+\mathrm{e}$, em que y é o vetor de dados, $\mathrm{r}$ é o vetor dos efeitos de repetição (fixos) somados à média geral, a é o vetor dos efeitos genéticos aditivos individuais (aleatórios), p é o vetor dos efeitos de parcela (aleatórios), e é o vetor de erros ou resíduos (aleatórios); $\mathrm{X}, \mathrm{Z}$ e W são as matrizes de incidência de $\mathrm{r}$, a e p, respectivamente. Para utilizar o modelo proposto, foi preciso presumir que o teste é composto por progênies de meios-irmãos originadas de polinização aberta. O teste de significância foi realizado com o cálculo do LTR (teste da razão de verossimilhança), que é obtido pela diferença entre as deviances para modelos sem e com o efeito a ser testado (progênie e parcela), usando-se o teste de qui-quadrado.

As estimativas de variâncias e parâmetros genéticos obtidas pelo programa são:

a) Herdabilidade individual no sentido restrito:

b) Herdabilidade da média de progênies:

$$
h_{a}^{2}=\frac{\sigma_{a}^{2}}{\sigma_{f}^{2}}
$$

$$
h_{m p}^{2}=\frac{\left(\frac{1}{4}\right) \sigma_{a}^{2}}{\left(\frac{1}{4}\right) \sigma_{a}^{2}+\frac{\sigma_{c}^{2}}{r}+\frac{\left(0,75 \sigma_{a}^{2}+\sigma_{\varepsilon}^{2}\right)}{n r}}
$$

c) Herdabilidade aditiva dentro de parcela:

d) Acurácia da seleção de progênies:

$$
h_{a d}^{2}=\frac{0,75 \sigma_{a}^{2}}{0,75 \sigma_{a}^{2}+\sigma_{a}^{2}}
$$

$$
r_{\mathrm{a} a}=\sqrt{h_{m}^{2}}
$$

e) Coeficiente de variação genético entre indivíduos:

$$
C V_{g i}(\%)=\frac{\sqrt{\sigma_{a}^{2}}}{\mu} 100
$$

f) Coeficiente de variação genético entre progênies:

$$
C V_{g p}(\%)=\frac{\sqrt{0,25 \sigma_{a}^{2}}}{\mu} 100
$$

g) Coeficiente de variação experimental:

$$
C V_{\theta}(\%)=\frac{\sqrt{\left[\frac{0,75 \sigma_{\varepsilon}^{2}}{n}\right]}+\sigma_{c}^{2}}{\mu} 100
$$




\section{Estimativas do tamanho efetivo populacional, ganho esperado e correlação}

Também foram estimados o tamanho efetivo populacional $\left(N_{e}\right)$, a diversidade genética $(D)$, o ganho $G_{s}(\%)$ e a eficiência $E f(\%)$ da seleção:

O tamanho efetivo populacional é daḑo por: $N_{\varepsilon}=\left(4 . N_{f} \bar{k}_{f}\right) /\left[\bar{k}_{f}+3+\left(\hat{\sigma}_{k f}^{2} / \bar{k}_{f}\right)\right]$ em que: $N_{f}$ = número de progênies selecionadas; $\sigma_{k f}^{2}=$ variância do número de indivíduos selecionados por progênie; $\bar{k}_{f}=$ número médio de indivíduos selecionados por progênie;

A diversidade genética $(D)$ após a seleção: $D=N_{e f} / N_{f o}$, em que: $0<\mathrm{D} \leq 1$, sendo: $N_{f o}$ = número original de famílias; $N_{e f}=$ número efetivo de famílias selecionadas, sendo dado por: $N_{e f}=\left(\sum k_{f}\right)^{2} / \sum k_{f}^{2}$. Com estas estimativas foram propostas duas estratégias de seleção, tendo por base a altura, por apresentar maior herdabilidade individual, visando a conservação genética do material e a formação de uma população base para futuros programas de melhoramento: $i$ ) Seleção de 60\% dos 393 indivíduos sobreviventes (seleção individual) com base no valor genético individual conforme o ranqueamento decrescente BLUP`s. Deste modo, pode acontecer de selecionar vários indivíduos da mesma progênie e nenhum indivíduo de outra progênie; ii) Seleção de $60 \%$ dentro das progênies, o que corresponde a um total de 310 indivíduos selecionados, 25 progênies x 3 plantas por progênie x 5 repetições. A forma como essa estratégia foi escolhida priorizou que todas as progênies se mantivessem no pomar de sementes.

O fato da sobrevivência ter sido muito baixa, foi proposta uma intensidade de seleção mais branda para evitar perda de variabilidade genética por ser uma população de primeira geração. A seleção objetivou deixar um mesmo número de indivíduos por progênies.

Adicionalmente obteve-se as estimativas de correlações fenotípicas e genéticas entre os caracteres.

\section{RESULTADOS E DISCUSSÃO}

A sobrevivência foi de 63\%, o que demonstra adaptação da espécie na região de estudo, já que o estudo foi realizado 25 anos após o plantio. Diferenças significativas entre progênies foram obtidas somente para bifurcação e sobrevivência. As médias para altura, DAP, volume e bifurcação foram de $12,91 \mathrm{~m}, 17,34 \mathrm{~cm}, 0,41 \mathrm{~m}^{3}$ árvore ${ }^{-1}$ e 9,62, respectivamente (Tabela 1). Aguiar et al. (1992) observaram altura e DAP de 11,45 m e 13,30 cm, respectivamente em progênies de D. alata, aos 20 anos de idade na região de Jaboticabal-SP.

O desempenho em altura e diâmetro dessa população de Dipteryx alata é semelhante aos de outras espécies nativas que possuem crescimento mais lento e com maior densidade de madeira. Pode-se citar, como exemplo, Gallesia integrifolia aos 20 anos (FREITAS et al., 2008), Balfourodendron riedelianum aos 21 anos (SEBBENN et al., 2007) e Myracrodruon urundeuva aos 17 anos (FREITAS et al., 2007). Sabe-se que quanto maior a densidade básica de madeira a tendência é de um crescimento mais lento (TUNG et al., 2011), os mesmos autores citam como exemplo a Myracrodruon urundeuva que possui densidade de madeira de $0,75 \mathrm{~g} \mathrm{~cm}^{-3}$, em ensaio avaliado aos 24 anos de idade em Selvíria, MS, e o próprio D. alata com densidade de madeira de $1,1 \mathrm{~g} \mathrm{~cm}^{-3}$ (SANO et al., 2004). Isso sugere uma boa adaptação da espécie na região de Pederneiras, SP.

A espécie apresentou um desempenho dentro da média, um outro indicador de adaptação é a produção sementes viáveis. Observou que a população apresenta uma boa produção de sementes, como algumas regenerações dentro e no entorno do teste.

O coeficiente de determinação dos efeitos ambientais entre parcelas $\left(c_{\text {parc }}^{2}\right)$ foram considerados de baixa magnitude, $\leq 17 \%$ (Tabela 1). Logo há maior precisão experimental e baixa variabilidade ambiental no teste, o que contribui para obter estimativas de parâmetros genéticos mais acurados (RESENDE, 2002a). Zaruma (2014) também encontrou valor inferior a 10\% para DAP em progênies de D. alata com 9 anos de idade. Assim como Santos et al. (2014b); Gomes (2011), em indivíduos da mesma espécie aos 11,5 e 11 anos, respectivamente.

É possível verificar que os coeficientes de herdabilidade no sentido restrito e aditivo dentro de parcela para todos os caracteres foram menores em relação à herdabilidade média para o teste de progênies de $D$. alata. Esse resultado é semelhante ao encontrado em outras espécies do cerrado como em Myracrodruon urundeuva (SEBBENN; ETTORI, 2001), Ilex paraguariensis (COSTA et al. 2005), Astronium fraxinifolium (SOUZA et al. 2003) e Balfourodendron riedelianum (SIQUEIRA et al. 2000). As estimativas de herdabilidade individual no sentido restrito, que quantifica a proporção 
aditiva da variância genética que pode ser transmitida para a próxima geração, foram baixas para maioria dos caracteres, exceto para altura (Tabela 01). Esses resultados ainda indicam baixa possibilidade de ganhos genéticos mediante seleção dentro de progênies. Segundo Bernardo (2010), os ganhos esperados na seleção dependem diretamente da herdabilidade e intensidade de seleção.

Os coeficientes de herdabilidade apresentam variações conforme as idades das plantas. Até que haja um completo estabelecimento e equilíbrio no campo, pode haver influência do ambiente, tanto maior quanto menor na manifestação das características de crescimento (ETTORI et al., 2006). Isso pode ser comprovado a partir de estudo realizado por Gomes (2011) em que o autor observou aumento da herdabilidade em progênies de D. alata em relação à idade. Segundo o autor, as herdabilidades individuais no sentido restrito para altura e DAP variaram entre 0,03 e 0,56 e 0,03 e 0,51, respectivamente em relação ao período estudado que foi de 11 anos. Contudo, o presente trabalho apresentou herdabilidade individual de 0,25 aos 25 anos de idade para altura, número considerado mediano em relação aos demais estudos anteriores, o que pode significar pequena atuação do ambiente nessas progênies

A acurácia dos caracteres altura, DAP, volume e sobrevivência foi média enquanto que bifurcação foi baixa. Resende e Duarte (2007) afirmam que, a acurácia, permite medir o grau de confiança das estimativas obtidas por meio de experimento de teste de progênies. Para Resende (2002a), valores entre 0 e $25 \%$ são considerados baixos, 25 e $75 \%$ são bons, e acima de $75 \%$ são ótimos. Valores baixos de acurácia estão relacionados ao tipo de delineamento que foi usado para estabelecimento do experimento e à baixa sobrevivência das plantas. O aumento da acurácia é possível reduzindo o desvio padrão das estimativas, que ocorre quando se aumenta o número de amostras por progênies (repetições ou parcelas) (HANNRUP et al., 1998). Quanto maior acurácia, maior precisão da seleção, e, consequentemente, maior o ganho genético (RESENDE, 1995). Em relação à variabilidade genética, sua quantificação é feita pelo coeficiente de variação genética, que expressa a magnitude da variação genética em relação à média do caráter (RESENDE et al., 2007). No presente trabalho os resultados foram considerados bons para altura $(12,65 \%)$, DAP $(10,94 \%)$ e sobrevivência $(22,68 \%)$, exceto para bifurcação (1,84\%), uma vez que, segundo Sebbenn et al. (1998), o coeficiente de variação genética maior que $7 \%$ pode ser indicativo de indivíduos superiores dentro das progênies e a possibilidade de se obterem ganhos com a seleção.

Em contrapartida, em teste de progênie da mesma espécie, Gomes (2011) obteve valores de coeficiente de variação genética em nível de indivíduo maiores que no presente trabalho entre 20,7\% a $24,0 \%$ e $23,61 \%$ a $35,13 \%$, respectivamente para altura e DAP com avaliações variando entre o primeiro e o décimo primeiro ano após o plantio. Zaruma (2014) também encontrou resultados maiores em progênies de D. alata aos 9 anos de idade (22,8 e 23,4\%, respectivamente para altura e DAP). Ettori et al. (1999) e Freitas et al. (2006) explicam que o baixo valor obtido nos resultados pode ser devido ao pequeno número de progênies testadas ou a pouca variabilidade existente.

Para a maioria dos caracteres, os coeficientes de variação experimental foram baixos (Tabela 1). Os resultados indicam que o controle experimental foi satisfatório, e pode-se esperar boa precisão nas estimativas de parâmetros genéticos. Valores de CVe entre 10 e 20\% podem ser considerados baixos para experimentos onde ocorre competição entre plantas (PIMENTEL-GOMES; GARCIA, 2002). Vale ressaltar, também, que esse parâmetro é afetado pelo tipo de caráter mensurado. Sebbenn et al. (2004) e Berti et al. (2011) afirmaram que, por exemplo, volume real ou cilíndrico de árvores em geral possuem valores acima de 40\%, corroborando com o presente trabalho. Valores semelhantes aos do presente trabalho foram obtidos por Rocha et al. (2009) e Zaruma (2014) para D. alata em outras localidades.

Na Tabela 2, observam-se os resultados obtidos na seleção utilizando-se o índice de BLUP, no qual são destacados as progênies e o número de indivíduos selecionados por progênie. Para Pereira e Vencovsky (1998), esse método tem como vantagem reduzir o peso dado à média geral de progênies. A estratégia $i$ de seleção individual com base nos valores genéticos das árvores sobreviventes, conforme o ranqueamento decrescente dos BLUPs, gerou ganhos genéticos satisfatórios com a seleção de 236 árvores (Tabela 2). Das 25 progênies de D. alata, 23 estão presentes nesse ranking, sendo que, em média, foram selecionados 10 indivíduos por progênie, o que corresponde a uma diversidade genética (D̂) de $69,0 \%$. Em relação ao tamanho efetivo $\left(N_{e}\right)$, após o desbaste, observou-se que nessa população de 236 plantas equivalem ao $N_{e}$ de $41,78(17,7 \%)$ e um ganho de seleção $\left(G_{s}\right)$ de $4,2 \%$. 
Pagliarini et al. - Variação genética em caracteres de crescimento em progênies de Dipteryx alata Vog.

Tabela 1. Estimativas de parâmetros genéticos para caracteres de crescimento em progênies de Dipteryx alata aos 25 anos de idade em Pederneiras, SP.

Table 1. Estimates of genetic parameters for growth traits at 25 years of age Dipteryx alata progenies to in Pederneiras, São Paulo State, Brazil.

\begin{tabular}{lccccc}
\hline Estimativas de Parâmetros & $\begin{array}{c}\text { Altura } \\
\mathbf{m}\end{array}$ & $\begin{array}{c}\text { DAP } \\
\mathbf{c m}\end{array}$ & $\begin{array}{c}\text { Volume } \\
\mathbf{m}^{\mathbf{3}} \text { árv }^{-1}\end{array}$ & $\begin{array}{c}\text { Bifurcação } \\
\%\end{array}$ & $\begin{array}{c}\text { Sobrevivência } \\
\%\end{array}$ \\
\hline$h^{2}{ }_{a}$ & $0,25( \pm 0,14)$ & $0,06( \pm 0,07)$ & $0,04( \pm 0,06)$ & $0,01( \pm 0,02)$ & $0,09( \pm 0,07)$ \\
$h^{2}{ }_{a j}$ & 0,27 & 0,06 & 0,04 & 0,01 & 0,10 \\
$h^{2}{ }_{m p}$ & 0,54 & 0,27 & 0,21 & 0,04 & 0,25 \\
$h^{2}{ }_{a d}$ & 0,22 & 0,05 & 0,03 & 0,01 & 0,08 \\
$C^{2}{ }_{p a r c}$ & 0,10 & 0,02 & 0,01 & 0,12 & 0,17 \\
$r_{a a}$ & 0,73 & 0,52 & 0,46 & 0,20 & 0,50 \\
$C V_{g i}(\%)$ & 12,65 & 10,94 & 19,06 & 1,84 & 22,68 \\
$C V_{g p}(\%)$ & 6,32 & 5,47 & 9,53 & 0,92 & 11,34 \\
$C V_{e}(\%)$ & 13,03 & 20,26 & 41,38 & 10,72 & 44,50 \\
$L R T_{\text {Progenies }}$ & $3,57^{\text {ns }}$ & $0,47^{\text {ns }}$ & $0,31^{\text {ns }}$ & $10,02^{* *}$ & $0,80^{\text {ns }}$ \\
$L R T_{\text {Parcelas }}$ & $3,53^{\text {ns }}$ & $0,10^{\text {ns }}$ & $0,10^{\text {ns }}$ & $15,00^{* *}$ & $24,45^{* *}$ \\
$\mu$
\end{tabular}

Herdabilidade individual no sentido restrito: $\mathrm{h}^{2} ;$ Herdabilidade individual ajustados para efeito de parcelas: $\mathrm{h}^{2} ;$; Herdabilidade média de progênies: $\mathrm{h}^{2}{ }_{\mathrm{mp}}$; Herdabilidade aditiva dentro de parcela: $\mathrm{h}^{2}{ }_{\text {ac }}$; Coeficiente de determinação do efeito de parcela: $\mathrm{C}^{2}$;arc ; Acurácia da seleção de progênie: $r_{\text {aj }}$; Coeficiente de variação genética individual: $\mathrm{CVgi}(\%)$; Coeficiente de variação genético entre progênies: $\mathrm{CVgp}$ (\%); Coeficiente de variação experimental: CVe (\%); Média geral: $\mu$; teste da razão de verossimilhança: LRT; Qui-quadrado tabelado: 3,84 e 6,63 para os níveis de significância de $5 \%$ (*) e 1\% (**), respectivamente. ${ }^{\text {ns }}$ Não significativo.

Na estratégia $i$ foi proposto selecionar $60 \%$ dos indivíduos dentro da progênie. Porém, em função de falhas existentes no teste verificou-se que seria possível manter apenas 310 árvores $(82,67 \%)$ das 375 plantas propostas anteriormente ( 25 progênies x 5 blocos x 3 plantas/bloco). Com a manutenção de todas as progênies a diversidade genética foi de $96,0 \%$, valor maior que à estratégia anterior (59\%). O $N_{e}$ obtido foi de 74,97 e um ganho com a seleção inferior a proposta anterior $(0,2 \%)$. A seleção der três plantas por bloco implica em desconsiderar outros indivíduos com valores genéticos superiores, e consequentemente, obter menor estimativa de ganho genético.

Na primeira estratégia, não há uma distribuição equitativa dos indivíduos selecionados nas famílias, e por consequência obtém-se estimativa menor do tamanho efetivo. Essa estratégia tem como como a proposta primordial de conservação genética, essa opção de seleção seria um desbaste de limpeza retirando do teste os indivíduos de menor desempenho, bem como para manter o mesmo número de indivíduos de cada progênies, já que números diferentes pode afetar o Ne em gerações subsequentes. Moraes (2001) afirma que ao delimitar o valor máximo de indivíduos selecionados por progênie, como aconteceu na segunda estratégia, consegue-se aumentar o número de progênies selecionadas, mantém-se o tamanho efetivo populacional adequado, além, de reter diversidade genética. Porém, maior ganho de seleção foi obtido na primeira estratégia em detrimento da segunda.

Estimativas de ganhos genéticos na seleção ocorrem, principalmente, em função da intensidade de seleção aplicada, da variação genética e da herdabilidade existentes (VENCOVSKY; BARRIGA, 1992). Dentre esses fatores, o melhorista florestal pode controlar apenas a intensidade de seleção, já que os outros podem facilmente ser alterados pela idade da planta e o ambiente. As estratégias de seleção devem ser escolhidas de forma consciente, pois, altas intensidades de seleção, por exemplo, podem ser arriscadas já que podem levar à eliminação ou perda precoce de alelos responsáveis por outros caracteres (KAGEYAMA; VENCOVSKY, 1983).

Como a espécie é plantada tanto para produção de madeira quanto para produção de sementes, a estimativa da variação genética de caracteres relacionados à produção de frutos, como a dimensão da copa, o número de bifurcações do fuste, número de frutos, etc, deveriam ser considerados antes de realizar desbaste seletivo.

Os coeficientes de correlação genética (Tabela 3) foram positivos e significativos para todos os pares de caracteres avaliados, variando de 0,05 a 0,94, sendo que o menor foi em relação Altura-Bifurcação e a maior em relação DAP-Volume. Segundo Sant'Ana et al. (2013), quando a seleção é efetuada em caracteres que possuam correlação positiva e de alta magnitude, pode-se esperar alta resposta correlacionada ocorrendo uma vantagem na seleção. Assim, para o presente trabalho, essa resposta pode ser encontrada na correlação entre DAP e volume. Dessa forma, se DAP ou volume 
forem adotados como características de seleção, não existe correlação genética desfavorável, o que torna o processo seletivo mais simples, já que ao modificar um caráter a tendência é o que o outro correlacionado também se modifique no mesmo sentido. Zaruma (2014) estudando progênies de D. alata, aos nove anos de idade, observou maior correlação entre DAP e altura enquanto que no presente trabalho a maior correlação foi encontrada entre DAP e Volume. Correlação positiva e significativa entre os caracteres facilita o melhoramento da população, principalmente quando se tem de considerar mais de um caráter no processo de seleção.

Tabela 2. Estratégias de seleção para o caráter altura em um teste de progênies de Dipteryx alata, aos 25 anos de idade, utilizando o índice multi-efeitos, em Pederneiras, SP.

Table 2. Selection strategies for height at 25 years of age Dipteryx alata progenies test using the multi-effect index in Pederneiras, São Paulo State Brazil.

\begin{tabular}{|c|c|c|c|c|}
\hline \multicolumn{3}{|c|}{ Estratégia a } & \multicolumn{2}{|c|}{ Estratégia $b$} \\
\hline & Prog & $k_{f}$ & Prog & $k_{f}$ \\
\hline & 1 & 8 & 1 & 14 \\
\hline & 2 & 18 & 2 & 15 \\
\hline & 3 & 10 & 3 & 15 \\
\hline & 4 & 13 & 4 & 13 \\
\hline & 5 & 15 & 5 & 14 \\
\hline & 6 & 14 & 6 & 12 \\
\hline & 7 & 2 & 7 & 12 \\
\hline & 8 & 2 & 8 & 14 \\
\hline & 9 & 14 & 9 & 12 \\
\hline & 10 & 14 & 10 & 12 \\
\hline & 11 & 7 & 11 & 9 \\
\hline & 12 & 19 & 12 & 15 \\
\hline & 13 & 13 & 13 & 15 \\
\hline & 14 & 9 & 14 & 9 \\
\hline & 15 & 8 & 15 & 13 \\
\hline & 16 & 2 & 16 & 10 \\
\hline & 18 & 8 & 17 & 10 \\
\hline & 19 & 4 & 18 & 14 \\
\hline & 21 & 7 & 19 & 8 \\
\hline & 22 & 17 & 20 & 15 \\
\hline & 23 & 6 & 21 & 11 \\
\hline & 24 & 24 & 22 & 14 \\
\hline \multirow{3}{*}{\multicolumn{2}{|c|}{25}} & 2 & 23 & 13 \\
\hline & & & 24 & 15 \\
\hline & & & 25 & 6 \\
\hline$N$ & & 236 & & 310 \\
\hline$N_{f o}$ & & 25 & & 25 \\
\hline$N_{f}$ & & 23 & & 25 \\
\hline $\bar{k}_{f}$ & & 10,26 & & 12,40 \\
\hline$\hat{\sigma}_{k f}^{2}$ & & 37,20 & & 6,33 \\
\hline$N_{e}$ & & 41,78 & & 74,97 \\
\hline$I M E_{a}$ & & 0,62 & & 0,02 \\
\hline$G_{s}(\%)$ & & 4,19 & & 0,16 \\
\hline$\hat{\mathrm{D}}$ & & 0,69 & & 0,96 \\
\hline$\mu$ & & 14,79 & & 12,95 \\
\hline
\end{tabular}

Número de indivíduos selecionados $(N)$; número de progênies originais $\left(N_{f 0}\right)$ número de progênie selecionadas $\left(N_{f}\right)$; número de indivíduos selecionados por progênie $\left(\bar{k}_{f}\right)$; variância do número de indivíduos selecionados por progênie $\left(\hat{\sigma}_{k f}^{2}\right)$; tamanho efetivo $\left(N_{e}\right)$; a: efeito genético aditivo $=I M E_{a}$ : Índice Multi-efeito; ganho na seleção $\left(G_{s}(\%)\right)$; diversidade genética $(\hat{D})$; média geral $(\mu)$.

As estimativas das correlações fenotípicas apresentaram valores significativos sendo positivos entre caracteres de crescimento (altura e DAP) e negativos entre bifurcação, altura e DAP (Tabela 3). A seleção indireta será eficiente nos dois casos, visto que um número menor de bifurcação é desejado. As associações positivas entre os caracteres indicam que a seleção em um caráter pode trazer ganhos indiretos em outro, em especial entre volume e DAP que apresentaram resultados de alta magnitude e positivos $(0,95)$. 
Pagliarini et al. - Variação genética em caracteres de crescimento em progênies de Dipteryx alata Vog.

Tabela 3. Estimativas das correlações genéticas $\left(r_{q}=\right.$ acima da diagonal) e fenotípicas $\left(r_{f}=\right.$ abaixo da diagonal) entre os caracteres silviculturais em progểnies de Dipteryx alata aos 25 anos de idade em Pederneiras, SP.

Table 3. Estimates of genetic correlations $\left(r_{g}=\right.$ above the diagonal) and phenotypic $\left(r_{f}=\right.$ below the diagonal) between silvicultural traits at 25 years of age Dipteryx alata progenies in Pederneiras, São Paulo State, Brazil.

\begin{tabular}{lcccc}
\hline & \multicolumn{3}{c}{ Caracteres } \\
\cline { 2 - 5 } Caracteres & Altura & DAP & Volume & Bifurcação \\
\hline Altura & - & $0,78^{* *}$ & $0,67^{* *}$ & $0,05^{* *}$ \\
DAP & $0,70^{* *}$ & - & $0,94^{* *}$ & $0,34^{* *}$ \\
Volume & $0,63^{* *}$ & $0,95^{* *}$ & - & $0,40^{* *}$ \\
Bifurcação & $-0,45^{* *}$ & $-0,60^{* *}$ & $-0,58^{* *}$ & - \\
\hline
\end{tabular}

*** $(\mathrm{p}<0,0 \mathrm{I})$ pelo teste $\mathrm{t}$.

\section{CONCLUSÕES}

Existe variação genética entre progênies de Dipteryx alata para todos os caracteres exceto para sobrevivência.

O controle genético dos caracteres é alto em nível de média de progênies para altura, médio para DAP, volume de madeira e sobrevivência e baixo para bifurcação.

A seleção individual é indicada para obter-se maior ganho genético e a seleção dentro de progênies para manter maior variabilidade genética nas sementes produzidas, sendo a segunda estratégia a mais indicada para formação do pomar de sementes por mudas por se tratar de um teste de primeiro ciclo de melhoramento e necessidade de manter variação genética.

Existe correlação genética e fenotípica de média a alta e positiva entre altura, DAP e volume de madeira e a seleção indireta é possível.

\section{REFERÊNCIAS BIBLIOGRÁFICAS}

AGUIAR, I. B; VALERI, S. V.; ISMAEL, L. J.; ALHO, D. R. Efeitos do espaçamento no desenvolvimento de Dipteryx alata Vog. em Jaboticabal-SP, até a idade de 20 anos. Revista Instituto Florestal, São Paulo, v. 4, n. 2, p. 570-572. 1992.

BERNARDO, R. Breeding for quantitative traits in plants. Woodbury: Stemma, 2010. 400 p.

BERTI, C. L. F.; FREITAS, M. L. M.; ZANATTO, A. C. S.; MORAIS, E.; MORAES, M. L. T.; SEBBENN, A. M. Variação genética, herdabilidade e ganhos na seleção para caracteres de crescimento e forma em testes de progênies de polinização aberta de Eucalyptus cloeziana. Revista do Instituto Florestal, São Paulo, v. 23, p. 13-26, 2011.

BORLAUG, N. E. Feeding a world of 10 billion people: themiracle ahead. In: BAILEY, R. (Ed.). Global warming and other eco-myths. Roseville: Competitive Enterprise Institute, 2002. p. 29-60.

BOTEZELLI, L.; DAVIDE, A. C.; MALAVASI, M. M. Características dos frutos e sementes de quatros procedências de Dipteryx alata VOGEL (BARU). Cerne, Lavras, v. 6, n. 1, p. 121-127, 2000.

CLEMENT, C. R. Melhoramento de espécies nativas. In: NASS, L. L.; VALOIS, A. C. C.; MELO, I. S.; VALADARESINGLIS, M. C. Recursos genéticos \& melhoramento: plantas. Rondonópolis: Fundação MT, 2001. p. 423-441.

CLEMENT, C. R.; LLERAS PÉREZ, E.; VAN LEEUWEN, J. Opotencial das palmeiras tropicais no Brasil: acertos e fracassosdas últimas décadas. Agrociencia, Montevideu, v. 9, n. 1-2, p.67-71, 2005.

COSTA, R. B.; RESENDE, M. D. V.; FERREIRA, J. S.; CONTINI, A. Z.; REGO, F. L. H.; ROA, R. A. R.; MARTINS, W. J. Avaliação genética de progênies de Leucaena leucocephala [(Lam.) De Wit] em áreas da reserva indígena, em Caarapó, MS. Floresta e Ambiente, Seropédica, v. 12, n. 1, p. 15-21, 2005. 
CZEDER, L. P. Composição nutricional e qualidade proteica da amêndoa de baru (Dipteryx alata Vog.) de plantas de três regiões do cerrado do estado de Goiás. 2009. 54 p. Dissertação (Mestrado em Ciência e Tecnologia de Alimentos) - Escola de Agronomia e Engenharia de Alimentos, Universidade Federal de Goiás, Goiânia, 2009.

ETTORI, L. C.; FIGLIOLIA, M. B.; SATO, A. S. Conservação ex situ dos recursos genéticos de espécies florestais nativas: situação atual no Instituto Florestal. In: HIGA, A. R.; SILVA, L. D. (Coord.). Pomar de sementes de espécies florestais nativas. Curitiba: FUPEF, 2006. p. 203-225.

ETTORI, L. C.; SIQUEIRA, A. C. M. F.; ZANATTO, A. C S.; VILAS BOAS, O. Variabilidade genética em duas populações de Cordia trichotoma. Revista do Instituto Florestal, São Paulo, v. 11, n. 2, p. 179-187, 1999.

FREITAS, M. L. M.; SEBBENN, A. M.; MORAIS, E.; ZANATTO, A. C. S.; VERARDI, C. K.; PINHEIRO, A. N. Parâmetros genéticos em progênies de polinização aberta de Cordia trichotoma (Vell.) ex Steud. Revista do Instituto Florestal, São Paulo, v. 18, n. único, p. 95-102, 2006.

FREITAS, M. L. M.; SEBBENN, A. M.; ZANATTO, A. C. S.; MORAES, E. Pomar de sementes a partir da seleção dentro de teste progênies de Myracrodruon urundeuva. Revista do Instituto Florestal, São Paulo, v. 19, n. 2, p. 65-72, 2007.

FREITAS, M. L. M.; SEBBENN, A. M.; ZANATTO, A. C. S.; MORAES, E.; MORAES, M. A. Variação genética para caracteres quantitativos em população de Gallesia intregrifollia. Revista do Instituto Florestal, São Paulo, v. 20, n. 2, p. 165-173, 2008.

GOMES, J. E. Variabilidade genética e correlações juvenil - adulto de baru (Dipteryx alata Vog.) no município de Brasilândia- MG. 2011. 101 p. Tese (Doutorado em Ciências Florestais) - Faculdade de Ciências Agronômicas, Universidade Estadual Paulista "Júlio de Mesquita Filho", Botucatu, 2011.

HANNRUP, B.; WILHELMSSON, L.; DANELL, O. Time trends for genetic parameters of wood density and growth traits in Pinus sylvestris L. Silvae Genetica, Frankfurt, v. 47, n. 4, p. 214-219, 1998.

KAGEYAMA, P. Y.; VENCOVSKY, R. Variação genética em progênies de uma população de Eucalyptus grandis (Hill) Maiden. IPEF, Piracicaba, v. 24, p. 9-26, 1983.

KUBOTA, T. Y. K.; MORAES, M. A.; SILVA, E. C. B.; PUPIN, S.; AGUIAR, A. V.; MORAES, M. L. T.; FREITAS, M. L. M.; SATO, A. S.; MACHADO, J. A. R.; SEBBENN, A. M. Variabilidade genética para caracteres silviculturais em progênies de polinização aberta de Balfourodendron riedelianum (Engler). Scientia Forestalis, Piracicaba, v. 43, n. 106, p. 01-08, 2015.

MITTERMEIER, R. A. Biodiversity Hotspots Revisited, Conservation International, Cidade do México: CEMEX, 2004. 390 p.

MORAES, M. L. T. Variação genética e aplicação da análise multivariada em progênies de Pinus caribaea var. hondurensis Barret e Golfari. 2001. 124 p. Tese (Livre Docência). Faculdade de Engenharia. Universidade Estadual Paulista. Ilha Solteira, 2001.

MYERS, N.; MITTERMEIER, R. A.; MITTERMEIER, C. G.; FONSECA, G. A. B.; KENT, J. Biodiversity hotspots for conservation priorities. Nature, Nova Iorque, v. 403, p. 853-858, 2000.

OLIVEIRA, A. N. Variabilidade genética entre e dentro de procedências de baru (Dipteryx alata Vog.). 1998. 80 p. Dissertação (Mestrado em Engenharia Florestal) - Universidade Federal de Lavras, Lavras, 1998.

PEREIRA, M. B.; VENCOVSKY, R. Limites da seleção recorrente. I. Fatores que afetam o acréscimo das frequências alélicas. Pesquisa Agropecuária Brasileira, Brasília, v. 23, n. 7, p. 768-780, 1998. 
Pagliarini et al. - Variação genética em caracteres de crescimento em progênies de Dipteryx alata Vog.

PIMENTEL-GOMES, F.; GARCIA, C. H. Estatística aplicada a experimentos agronômicos e florestais: exposição com exemplos e orientações para uso de aplicativos. Piracicaba: FEALQ, 2002. 309 p.

RESENDE, M. D. V. Delineamento de experimentos de seleção para maximização da acurácia seletiva e do progresso genético. Revista Árvore, Viçosa, v. 19, n. 4, p. 479-500, 1995.

RESENDE, M. D. V. Genética biométrica e estatística no melhoramento de plantas perenes. Brasília: EMBRAPA Informação Tecnológica, 2002a. 975 p.

RESENDE, M. D. V. Software Selegen-REML/BLUP. Curitiba: EMBRAPA Florestas, 2002b. 67 p. (Documento, 77)

RESENDE, M. D. V.; DUARTE, J. B. Precisão e controle experimental de qualidade em experimentos de avaliação de cultivares. Pesquisa Agropecuária Tropical, Goiânia, v. 37, n. 3, p. 182-194, set. 2007.

ROCHA, M. G. B.; ROCHA, D.; CLEMENTE, V. M. Teste de procedências e progênies de baru (Dipteryx alata Vogel). In: ROCHA, M. G. B. Melhoramento de espécies arbóreas nativas. Belo Horizonte: DDFS/Instituto Estadual de Florestas, 2002. p. 29-40.

ROCHA, R. B.; ROCHA, M. G. B.; SANTANA, R. C.; VIEIRA, A. H. Estimação de Parâmetros Genéticos e Seleção de Procedências e Famílias de Dipteryx alata Vogel (BARU) utilizando metodologia de REML-BLUP e E(QM). Cerne, Lavras, v. 15, n. 3, p. 331-338, 2009.

SANO, S. M.; RIBEIRO, J. F; BRITO, M. A. Baru: biologia e uso. Planaltina: Embrapa Cerrados, 2004.52 p. (Documentos, 116)

SANT'ANA, V. Z.; FREITAS, M. L. M.; MORAES, M. L. T.; ZANATA, M.; ZANATTO, A. C. S.; MORAES, M. A.; SEBBENN, A. M. Parâmetros genéticos em progênies de polinização aberta de Enterolobium contortisiliquum (Vell.) Morong em Luiz Antonio, SP, Brasil. Hoehnea, São Paulo, v. 40, n.3, p. 515-520, 2013.

SANTOS, A. M.; ROSADO, S. C. S.; OLIVEIRA, A. N. Estimation of genetic parameters and verification of early selection efficiency in baru (Dipteryx alata). Crop Breeding and Applied Biotechnology, Viçosa, v. 14, n. 4, p. 238-243, 2014b.

SANTOS, M. A.; BARBIERI, A. F.; MACHADO, C. J.; CARVALHO, J. A. Minas Gerais e o marco inicial da ocupação do cerrado brasileiro: o papel do estado. Redes, Santa Cruz do Sol, v. 19, n. 1, p. 261-275, 2014a.

SEBBENN, A. M.; ETTORI, L. C. Conservação genética ex situ de Esenbeckia leiocarpa, Myracrodruon urundeuva e Peltophorium dubium em teste de progênies misto. Revista do Instituto Florestal, São Paulo, v. 13, n. 2, p. 201-211, 2001.

SEBBENN, A. M.; FREITAS, M. L. M; ZANATTO, A. C. S.; MORAES, E.; MORAES, M. A. Conservação ex situ e pomar de sementes em banco de germoplasma de Balfourodendron riedelianum. Revista Instituto Florestal, São Paulo, v. 19, n. 2, p. 101-112, 2007.

SEBBENN, A. M.; PONTINHA, A. A. S.; FREITAS, S. A.; FREITAS, J. A. Variação genética em cinco procedências de Araucaria angustifólia (Bert.) O. Ktze. no sul do Estado de São Paulo. Revista do Instituto Florestal, São Paulo, v. 16, n. 2, p. 91-99, 2004.

SEBBENN, A. M.; SIQUEIRA, A. C. M. F.; KAGEYAMA, P. Y.; MACHADO, J. A. R. Parâmetros genéticos na conservação da cabreuva - Myroxylon peruiferum L.F. Allemão. Scientia Forestalis, Piracicaba. n. 53, p. 31-38, 1998. 
SIQUEIRA, A. C. M. F.; SEBBENN, A. M.; ETTORI, L. C.; NOGUEIRA, J. C. B. Variação genética entre e dentro de populações de Balfourodendron riedelianum (Engler) Engler para conservação ex situ. Revista do Instituto Florestal, São Paulo, v. 12, n. 2, p. 89-103, 2000.

SOUZA, C. S.; AGUIAR, A. V.; SILVA, A. M.; MORAES, M. L. T. Variação genética em progênies de Gonçalo Alves (Astronium fraxinifolium) em dois sistemas de plantio. Revista do Instituto Florestal, São Paulo, v. 15, n. 2, p. 137-145, 2003.

TAKEMOTO, E.; OKADA, I. A.; GARBELOTTI, M. L.; TAVARES, M.; AUED-PIMENTEL, S. Composição química da semente e do óleo de baru (Dipteryx alata Vog.) nativo do Município de Pirenópolis, Estado de Goiás. Revista Instituto Adolfo Lutz, São Paulo, v. 60, n. 2, p. 113-117, 2001.

TUNG, E. S. C.; FREITAS, M. L. M.; FLORSHEIM, S. M. B.; LIMA, I. L; LONGUI, E. L.; MORAES, M. L. T.; SANTOS, F. W.; SEBBENN, A. M. Variação, divergência e correlações genéticas entre caracteres silviculturais e densidade básica da madeira em progênies de Myracrodruon urundeuva (Engler) Fr. Allem. Revista Instituto Florestal, São Paulo, v. 23, n. 1, p. 1-12, 2011.

ZARUMA, D. U. G. Variações genéticas para caracteres silviculturais em progênies e procedências de Dipteryx alata Vogel. 2014. 53 p. Dissertação (Mestrado em Ciência Florestal). Universidade Estadual Paulista "Júlio de Mesquita Filho", Botucatu, 2014.

Recebido em 20/10/2015

Aceito para publicação em 23/03/2016

Sci. For., Piracicaba, v. 44, n. 112, p. 925-935, dez. 2016

DOI: dx.doi.org/10.18671/scifor.v44n112.14 
